\title{
Standardised PCR-based molecular epidemiology of tuberculosis
}

\author{
C. Allix-Béguec*,\#, P. Supply ${ }^{\star}$, M. Wanlin ${ }^{+}$, P. Bifani and M. Fauville-Dufaux*
}

ABSTRACT: A population-based molecular epidemiology investigation has been undertaken to
evaluate tuberculosis transmission and control in the Brussels-Capital Region (Belgium). All tuberculosis cases reported from January 2003 to December 2004 were investigated. In total, 536 Mycobacterium tuberculosis isolates ( $89 \%$ of culture-positive samples) were genotyped by the newly standardised 24 loci-based mycobacterial interspersed repetitive unit-variable number tandem-repeat typing, spoligotyping and IS6110 fingerprinting.

Of all the patients, $30 \%$ were grouped based on strain clusters, suggesting a transmission index of $20 \%$. An unsuspected outbreak entailing $\geqslant 23$ patients was evidenced by molecular typing analysis and confirmed by contact tracing. Foreign-born status accounted for $79 \%$ of the studied patients, including $\mathbf{3 7 . 9 \%}$ illegal immigrants and asylum seekers. Among foreign-born patients, asylum seekers and illegal immigrants were significantly less abundant in strain clusters than settled residents.

Tuberculosis in the Brussels-Capital Region is a bi-faceted problem, comprising both persisting recent transmission and "imported diseases". Molecular epidemiology based on real-time genotyping techniques has proven invaluable in better understanding tuberculosis transmission. However, it will most efficiently contribute to tuberculosis control when implemented in an integrated public health system.

\section{KEYWORDS: DNA fingerprinting, epidemiology, Mycobacterium tuberculosis, transmission}

I n Belgium, the evolution of tuberculosis (TB) incidence is similar to that recently observed in many other industrialised countries. TB morbidity and mortality has uniformly declined during the 20th century. A total of 1,128 and 1,226 new cases were reported to the Belgian Lung and Tuberculosis Association (BELTA) in 2003 and 2004, respectively (10.9 and 11.8 cases per 100,000 inhabitants in 2003 and 2004, respectively) [1, 2]. However, TB incidence in the Brussels-Capital Region is three times higher than in the rest of the country (358 and 342 new cases in 2003 and 2004, which correspond to an incidence of 36.1 and 34.2 per 100,000, respectively) [1, 2]. The overall incidence rate in this region has not declined in the same manner as in the rest of Belgium, owing to the impact of imported TB cases associated with the growing immigrant population. Indeed, the Brussels-Capital Region, which has hosted the headquarters of the European Union since 1958, is a cosmopolitan area of approximately 1 million inhabitants, in which the proportion of foreign residents has risen from $7 \%$ in 1961 to $26 \%$ of the population in 2004 [3]. This region hosts $30 \%$ of all foreign residents registered in Belgium, whereas it hosts only $10 \%$ of the country population [4]. Moreover, among the foreign-born population of the Brussels-Capital Region, reported TB incidence in 2004 was 99.5 cases per 100,000 persons, including asylum seekers and illegal residents (63.8 cases per 100,000 inhabitants when including only foreign-born residents), which is much higher than the incidence of 10.9 per 100,000 inhabitants reported in the Belgian-born population [2].

Mycobacterium tuberculosis complex DNA fingerprinting has proven to be an invaluable tool for TB control and monitoring as it enables identification of unsuspected transmission between strain-clustered patients and false-positive cultures [5-8]. However, only a few countries have managed to integrate molecular typing into their TB control strategy $[9,10]$. This is partly due to limitations of the available genotyping techniques. The gold standard method, restriction fragment length polymorphism (RFLP) using IS6110 as a probe, is laborious and requires weeks of culturing of the slow-growing M. tuberculosis [11]. PCRbased methods, such as spoligotyping [12] and typing based on 12 loci containing variable numbers of tandem repeats of mycobacterial interspersed repetitive units (MIRU-VNTRs) [13], are applicable to crude DNA extracts from early cultures and are thus much faster. However, they
AFFILIATIONS

*Laboratoire Tuberculose et Mycobactéries, Institut Pasteur Institut Scientifique de Santé Publique.

+Belgian Lung and Tuberculosis Association, Brussels, Belgium.

${ }^{\text {\#} G e n o s c r e e n, ~ a n d ~}$

- Laboratoire des Mécanismes Moléculaires de la Pathogenèse Bactérienne, INSERM U629, Institut Pasteur, Lille, France.

CORRESPONDENCE

M. Fauville-Dufaux

Laboratoire Tuberculose et

Mycobactéries

Institut Pasteur

Bruxelles (Institut Scientifique de Santé Publique)

rue Engeland 642

1180 Bruxelles

Belgium

Fax: 3223733281

E-mail: mfauville@pasteur.be

Received:

May 032007

Accepted after revision:

December 052007

\section{SUPPORT STATEMENT}

C. Allix-Béguec was a fellow of the Brussels-Capital Region. P. Supply is a researcher at the Centre National de la Recherche Scientifique (CNRS).

STATEMENT OF INTEREST A statement of interest for P. Supply can be found at www.erj.ersjournals.com/misc/ statements.shtml 
have less discriminatory power than IS6110-RFLP [14]. To circumvent these limitations, a newly optimised MIRU-VNTR technique has recently been proposed [15]. This system, based on 15 or 24 loci, showed a discriminatory power similar to that of IS6110-RFLP, as recently described [16].

In order to estimate the level and evaluate the control of TB transmission in the Brussels-Capital Region, this newly standardised high-resolution genotyping system was used to conduct a prospective population-based molecular epidemiology study from January 1, 2003 to December 31, 2004. Spoligotyping and IS6110-RFLP were also performed as complementary and control methods, respectively. DNA fingerprinting was then used to determine the genetic diversity and the magnitude of $M$. tuberculosis transmission rate in this cosmopolitan setting. Predictors of strain clustering were analysed and epidemiological links between patients were examined based on molecular results.

\section{MATERIAL AND METHODS}

\section{Study subjects}

From January 1, 2003 to December 31, 2004, 700 new TB cases were registered by BELTA in the Brussels-Capital Region. Positive cultures were obtained from 603 patients, of whom 536 cases $(89 \%$ of culture-positive patients) were genotyped. The remaining 164 (out of 700 ) patients were not typed due to culture-negative samples $(n=97)$, culture unavailability $(n=64)$, or contamination by other microorganisms $(n=3)$. Six (out of 536) cases were additionally excluded because of probable laboratory cross-contamination, as inferred by genotyping.

\section{Study design}

Sample isolation and identification were carried out in one of five qualified hospitals of the Brussels-Capital Region and at the Pasteur Institute (Brussels, Belgium). As soon as M. tuberculosis cultures were available, they were immediately genotyped by PCR methods at the Tuberculosis and Mycobacteria Reference Center, Pasteur Institute.

Demographic and clinical data, collected by clinicians and nurses using a standardised questionnaire, was obtained from the Belgium Tuberculosis Register. Information was obtained on sex, date and country of birth, nationality, resident status, present address, recent contact $(<2$ yrs) with a TB patient and socioeconomic status. For the latter parameter, patients were considered underprivileged when being identified as homeless and/or having less than one meal per day.

\section{Methods}

All samples were subjected to spoligotyping and standardised MIRU-VNTR analysis based on 24 loci, while $48 \%$ were further typed by RFLP [7-9]. A strain cluster was defined as two or more patients infected by strains having identical spoligotypes and MIRU-VNTR patterns. IS6110-RFLP, spoligotyping and MIRUVNTR profiles were analysed using the Bionumerics package (Applied Maths, St-Martin-Latem, Belgium). Dendrograms were generated using the dice coefficient and the unweighted pair group method with arithmetic averages. Assuming that one patient from each strain cluster corresponded to the index case at the origin of infection, strain-clustering rate (or recent transmission index (RTI)) was calculated as follows:

$$
\mathrm{RTI}_{\mathrm{n}-1}=\left(\mathrm{n}_{\mathrm{c}}-\mathrm{c}\right) / \mathrm{n}
$$

where $n_{c}$ is the total number of strain-clustered cases, $c$ is the number of strain-clusters, and $n$ is the total number of cases in the sample [5].

\section{Analysis}

The association between five factors selected a priori (sex, age, form of disease, region/country of birth (aggregated in three categories) and socioeconomic status indicator) and strainclustering was studied. Odds ratio (OR) was defined as the odds of being in a strain cluster relative to the odds of being infected by a unique isolate. Bivariate and multivariate ORs and $95 \%$ confidence intervals were estimated from logistic models. Interactions between country/region of birth and other selected factors were tested using the likelihood ratio test. Interaction involving one predictor and country/region of birth was found to be significant. The ORs for this factor within each category of country/region of birth was estimated by exponentiation of an appropriately calculated logit difference. The p-values given beside adjusted ORs are those obtained with the Wald test [17].

\section{RESULTS}

\section{Cosmopolitan population}

Among the 530 patients included in the study, $61.3 \%$ were male and the median age was 34 yrs (table 1). Previous history of TB was registered for $10.8 \%$ of the patients. Drug resistance was diagnosed in $8.4 \%$ of the cases, while multidrug-resistant TB was only detected among foreign-born TB patients $(1.7 \%$ of all TB cases). A total of $45 \%$ of the patients had a contagious form of the disease. HIV-positive patients accounted for $10 \%$ of the cases, while $12 \%$ of the patients had another immunosuppressive disease or treatment. When considering lifestyle risk factors, 5.6, 1.7 and $3.6 \%$ of the patients were registered as alcohol abuser, prisoner and/or having occupational risk of TB, respectively. Moreover, almost half $(48.1 \%)$ of TB patients were considered underprivileged. In total, $78.8 \%$ of the study population were foreign-born. These patients originated from 61 different countries, of which the eight most frequently encountered were Morocco (28.2\%), Democratic Republic of the Congo (12.2\%), Turkey (6.1\%), Pakistan (4.1\%), Romania (3.3\%), Ecuador (3.1\%), Guinea (2.5\%) and Rwanda (2.5\%). All these countries, except Turkey, have a high TB incidence $(>100$ per 100,000 inhabitants). Among the foreign-born patients, $37.9 \%$ were asylum-seekers or illegal immigrants. The proportion of subjects involved in a strain cluster (see later) was $29.6 \%$.

\section{Strain-cluster analysis}

Out of the 530 patients, 157 were grouped into 53 strain clusters of two to 23 patients, resulting in a strain-clustering rate of $20 \%$. Given the resolution and the robustness of the combined spoligotype-MIRU-VNTR analysis [14], especially with this 24-loci-based MIRU-VNTR system, IS6110-RFLP was performed only on a subset $(n=256)$ of isolates, to further confirm strain clustering defined by MIRU-VNTR and spoligotyping. The mutual correspondence between the respective strain clusters was almost perfect [18].

Based on these genotyping results, TB patients were divided into two groups: patients grouped according to strain clusters and patients infected by an isolate with a unique pattern in the 


\section{TABLE 1 Sample description}

\begin{tabular}{|c|c|}
\hline & Sample proportion \\
\hline \multicolumn{2}{|l|}{ Demographics } \\
\hline Male & $61.3(325 / 530)$ \\
\hline Age yrs ${ }^{\#}$ & $34(25)$ \\
\hline $0-24$ & $22.1(117 / 530)$ \\
\hline $25-34$ & $29.2(155 / 530)$ \\
\hline $35-49$ & $22.5(119 / 530)$ \\
\hline$\geqslant 50$ & $26.2(139 / 530)$ \\
\hline \multicolumn{2}{|l|}{ TB characteristics } \\
\hline Previous history of TB & $10.8(48 / 445)$ \\
\hline Any resistance & $8.4(44 / 523)$ \\
\hline MDR & $1.7(9 / 523)$ \\
\hline \multicolumn{2}{|l|}{ Physiological risk factors } \\
\hline Pulmonary TB smear-positive & $45.0(227 / 505)$ \\
\hline HIV-positive & $10.0(47 / 472)$ \\
\hline Disease/treatment & $12.0(55 / 462)$ \\
\hline \multicolumn{2}{|l|}{ Lifestyle risk factors } \\
\hline Alcohol abuse & $5.6(25 / 450)$ \\
\hline Incarceration & $1.7(8 / 464)$ \\
\hline Underprivileged & $48.1(223 / 464)$ \\
\hline Occupational risk & $3.6(16 / 449)$ \\
\hline \multicolumn{2}{|l|}{ Country/region of birth } \\
\hline Belgium & $22.2(112 / 505)$ \\
\hline Western Europe & $5(25 / 505)$ \\
\hline Asia & $7.5(38 / 505)$ \\
\hline Africa unspecified & $12.7(64 / 505)$ \\
\hline South America & $4.8(24 / 505)$ \\
\hline Central and Eastern Europe & $7.5(38 / 505)$ \\
\hline Demographic Republic of Congo & $9.5(48 / 505)$ \\
\hline Middle East & $8.9(45 / 505)$ \\
\hline Morocco & $22(111 / 505)$ \\
\hline \multicolumn{2}{|l|}{ Residence status } \\
\hline Asylum seeker or illegal resident & $37.9(135 / 356)$ \\
\hline \multicolumn{2}{|l|}{ Recent transmission } \\
\hline Part of a strain cluster & $29.6(157 / 530)$ \\
\hline \multicolumn{2}{|c|}{$\begin{array}{l}\text { Sample proportions are given as number of patients with characteristic/total } \\
\text { number of patients with available data. TB: tuberculosis; MDR: multidrug resistant } \\
\text { (resistance to at least isoniazide and rifampicin). }{ }^{*}: \text { median (interquartile range) } \\
\text { : pneumoconiosis, neck-head cancer, leukaemia, Hodgkin, renal insufficiency } \\
\text { type-I diabetes, immunosuppressive treatment. }\end{array}$} \\
\hline
\end{tabular}

population. This molecular epidemiological data was then compared with epidemiological parameters classically considered as plausible risk factors for a patient to be part of a strain cluster, supposedly reflecting recent transmission. These parameters included sex, age, site of disease, socioeconomic status and country of birth (table 2). No significant differences between males and females and among age strata were observed. Concerning the site of disease, $\mathrm{p}$-value was close to significance threshold $(p=0.061)$. As interaction involving the socioeconomic indicator and country/region of birth was found to be significant $(p=0.004)$, the ORs (association with strain clustering) for these factors within each category of origin were estimated. The country/region of birth groups considered comprised patients from: Belgium and Western
Europe, Morocco and other origins, respectively, in order to enable statistical analysis with sufficient sample sizes. Among Belgian and Western European patients, being underprivileged was a strain-clustering predictor $(\mathrm{p}<0.001)$.

\section{Molecular guided identification of TB transmission}

Molecular analysis allowed for the identification of 53 strain clusters (157 patients). Combining molecular and demographic data revealed little cross-national TB transmission (fig. 1). The single three clusters comprising Belgian-born and foreign-born settled and unsettled immigrant patients (central area in fig. 1; $3 \mathrm{sc}$ ), including the largest strain cluster of 23 patients (10 Belgian, six Moroccan, two Polish, one Hungarian, one Luxembourgian, one Pakistani, one Portuguese and one Spanish patient), suggest extensive transmission. Of these 23 patients, 20 had pulmonary TB, 20 were male and one was 12 yrs old, while the mean \pm SD age was $44 \pm 15.6$ yrs. The outbreak strain had the very rare spoligotype 776000000000171 of the S-strain genotype in the worldwide spoligotype database (fig. 2) [19]. Contact tracing was possible for 12 patients but not for the others, due to change of address, illegal status or death. Links were confirmed in 11 out of 12 patients. Linked patients were relatives, friends, acquaintances of the same nationality or neighbours. In addition, within the infected families there were three culture-negative TB-diagnosed paediatric cases also suspected as belonging to this outbreak. The two other strain clusters grouping Belgian-born, settled and unsettled patients were one of six patients (four settled, one unsettled and one Belgian) and one of three patients (including a settled father and his Belgian-born son).

Among the 50 other and smaller strain clusters of two to six patients each (125 patients), five strain clusters comprised exclusively of Belgian-born patients $(n=13)$, while 24 consisted exclusively of foreign-born patients, either settled $(n=50)$ or unsettled ( $n=7$; fig. 1). Five strain clusters comprised foreignborn patients and their Belgian-born children (one asylum seeker and Belgian-born son; and four settled immigrants and their Belgian-born children). Other clusters consisting of Belgian-born and settled immigrants included one cluster of six patients (five Belgian-born patients and one settled resident).

\section{Suspected epidemiological links in small clusters}

Systematic contact tracing could not be carried out for patients of the 52 small strain clusters. However, when comparing information from routine epidemiological surveillance with strain-clustering detection, recent contact $(<2$ yrs $)$ with a TB patient was mentioned by $20.6 \%$ of Belgian-born and $23.6 \%$ of foreign-born strain-clustered patients; however, the proportion of missing data, $32.1 \%$ overall, was important for this variable. More detailed analyses of the other epidemiological data (including demographic and clinical data) showed that family transmission or social contact among patients with the same nationality took place for $\geqslant 40$ subjects distributed into 18 strain-clusters (fig. 2). For these patients, the median time between the detection of the source case and the secondary case was 22 days. Eight other strain clusters comprised patients with undetermined links living within a radius of $1 \mathrm{~km}$ from each other. For three of these strain clusters, some patients were infected by a strain genotype infrequently associated with their country/region of birth, in contrast with 


\begin{tabular}{|c|c|c|c|c|c|}
\hline Sex & & & & & 0.281 \\
\hline Male & 278 & 32.7 & $1.5(1.0-2.3)$ & $1.3(0.8-2.0)$ & \\
\hline Female & 169 & 24.9 & 1.0 & 1.0 & \\
\hline $0-24$ & 101 & 33.7 & $1.3(0.7-2.3)$ & $1.8(0.9-3.4)$ & \\
\hline $25-34$ & 137 & 26.3 & $0.9(0.5-1.6)$ & $1.4(0.7-2.7)$ & \\
\hline $35-49$ & 100 & 32.0 & $1.2(0.7-2.1)$ & $1.3(0.7-2.6)$ & \\
\hline$\geqslant 50$ & 18 & 28.4 & 1.0 & 1.0 & \\
\hline Site of disease & & & & & 0.061 \\
\hline Pulmonary smear-positive & 102 & 36.1 & $1.8(1.2-2.6)$ & $1.5(1.0-2.4)$ & \\
\hline Underprivileged & 48 & 64.6 & $6.2(2.7-14.2)$ & $5.6(2.4-13.1)$ & \\
\hline Not underprivileged & 66 & 22.7 & 1.0 & 1.0 & \\
\hline Morocco & & & & & 0.461 \\
\hline Underprivileged & 49 & 40.8 & $1.1(0.5-2.5)$ & $1.0(0.4-2.4)$ & \\
\hline Not underprivileged & 47 & 38.3 & 1.0 & 1.0 & \\
\hline Others & & & & & 0.414 \\
\hline Underprivileged & 121 & 21.5 & $1.1(0.6-2.1)$ & $1.0(0.6-2.0)$ & \\
\hline Not underprivileged & 116 & 19.8 & 1.0 & 1.0 & \\
\hline
\end{tabular}

Multilogistic regression analysis was carried out. The sample comprised a total of 447 subjects. OR: odds ratio; Cl: confidence interval

other patients originating from countries whereby these strain types are known to be prevalent. For these patients with possible links, the median time between the detection of the source case and the secondary cases was 275 days. No obvious link was found for 69 patients belonging to any of the 26 different remaining strain clusters.

\section{DISCUSSION}

The present study is a cross-sectional population-based survey conducted in a group of 530 culture-positive TB patients registered in Brussels during a 2-yr period (2003-2004). This group represents $89 \%$ of all of the bacteriologically confirmed cases, corresponding to $75 \%$ of all TB cases from the BrusselsCapital Region reported to the TB Register over the study period [1, 2]. Molecular analysis of this representative collection provides a snapshot of $\mathrm{TB}$ transmission in the European capital, and reveals some aspects with important implications for TB control. Notably, TB in the Brussels-Capital Region is a bi-faceted problem, resulting from largely unsuspected ongoing transmission and a mixture of recently imported cases from high-incidence countries.

The current authors used the recently standardised MIRUVNTR set [15] in combination with spoligotyping, which allowed tracking and rapid detection of strain clusters. The largest strain cluster $(n=23)$ comprised a strain of a characteristically exceptional S-spoligotype (see supplemental table in [19]), referred to as the S-Brussels. This strain was isolated from 10 Belgian-born and 13 foreign-born patients of mixed origin, suggesting recent transmission. Such recent transmission was confirmed after retrospective molecular-guided contact tracing in 11 out of 12 investigated cases. A noteworthy observation is that five and 13 additional TB patients were diagnosed with the same S-Brussels strain in the months preceding and following the period of the investigation, respectively, thus bringing the total number to 41 patients diagnosed with this strain within a 4yr period. Furthermore, this strain has been identified in a neighbouring region not included in the present study. These data strongly suggest that strain S-Brussels is being actively transmitted in the community. The identification by molecular typing of this unsuspected, large, still-expanding strain-cluster, in addition to other strain-clusters, further demonstrates ongoing TB transmission in the Brussels-Capital Region. The current authors hypothesise that the magnitude of ongoing TB transmission is probably similarly underestimated in numerous other metropolitan areas in developed countries with comparable characteristics, but where molecular epidemiology is not systematically applied.

Within the Brussels-Capital Region, ongoing transmission is significant as $30 \%$ of the isolates, corresponding mostly to autochthons and settled residents, were clustered by fingerprint analysis. This corresponds to a strain-clustering rate of $20 \%$ in close agreement with the few other studies in similar settings [20]. Sex inequalities in TB have been reported [21] but were not observed among strain-clustered patients in the present TB population. No differences were found among patients from different age strata when these strata were 


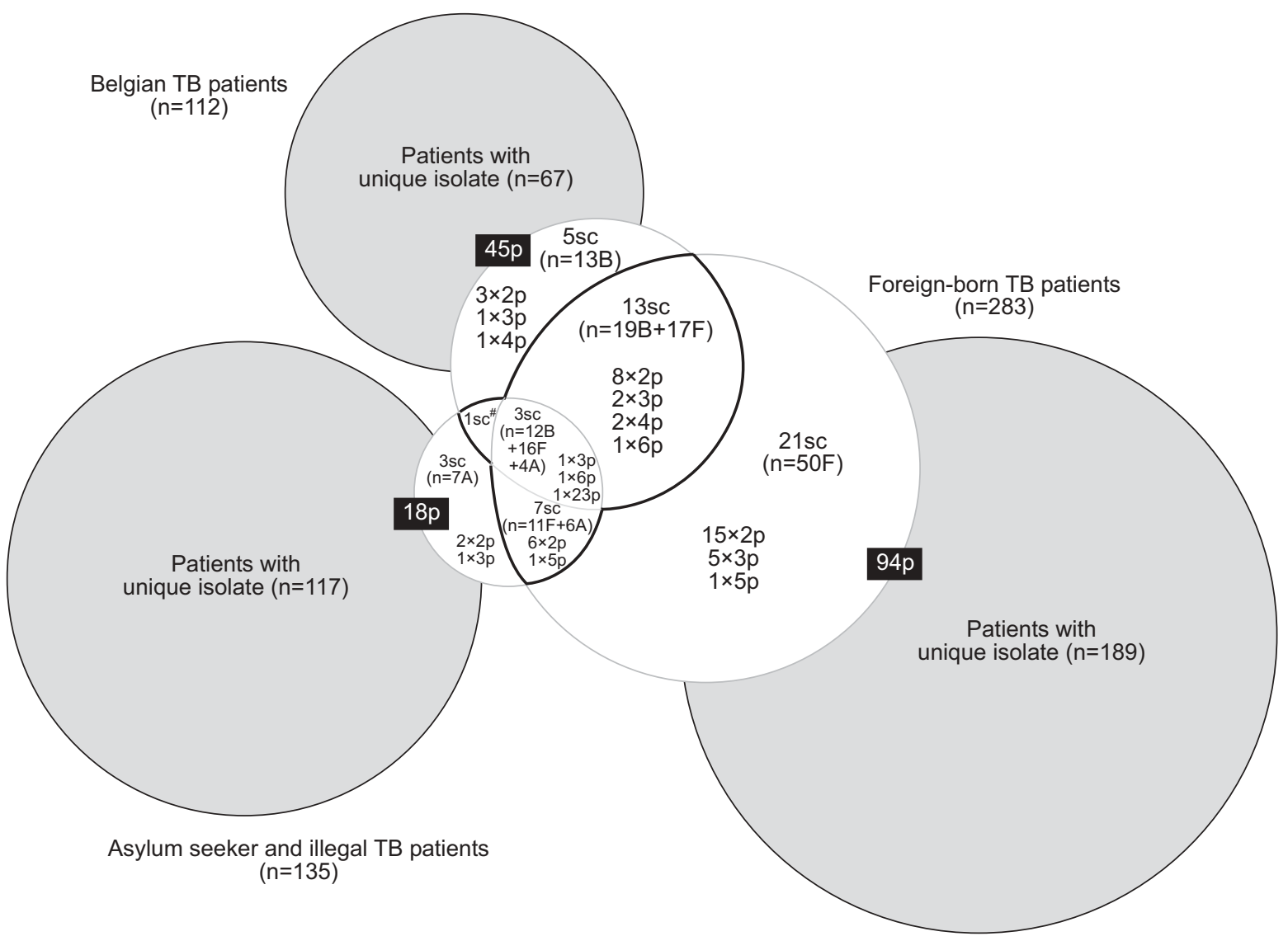

FIGURE 1. Study of tuberculosis (TB) transmission. Patients were classified according to their country/region of birth and resident status. Numbers and sizes of strain clusters and numbers of concerned patients (of different country/region of birth and status) are indicated in each section. 1 : patients infected by unique isolates; $\square$ : strainclustered (sc) patients. p: patient; B: Belgian-born patients; F: foreign-born patients; A: asylum seekers and illegal patients. ${ }^{*}$ : TB transmission from an asylum seeker parent to a Belgian-born child.

defined as age groups of roughly equal sizes. Nevertheless, consistent with the fact that younger patients are more likely to acquire TB from recent transmission, age became a predictor of strain-clustering when age strata of 15 yrs were defined, which resulted in age groups of very disparate sizes (data not shown). Moreover, even if the significance threshold was not reached for the site of disease, patients with a smear-positive pulmonary TB, i.e. patients with a contagious form of the disease, tended to be more often associated with strain clustering. When looking at country/region of origin and socioeconomic status, strain clustering was most frequently encountered in underprivileged Belgian and Western European-born patients, which is consistent with identified risk groups among this population. Contact tracing or analysis of available demographic data confirmed familial or social links for $\geqslant 51$ (32\%) out of 157 strain-clustered patients. Patients were found to live in close proximity in 25 (16\%) out of 157 additional strain-clustered cases of unknown epidemiological links.

According to the Belgian TB register, $77 \%$ of the active TB cases reported in the Brussels-Capital Region in 2003 and 2004 (79\% of the study population) are foreign-born $[1,2]$. In the present study, more than one-third of the foreign-born TB cases $(37.9 \%)$ were asylum seekers and illegal residents. The familial link observed in strain-clusters among settled immigrants and the limited strain-clustering in foreign-born unsettled patients suggest that they are imported cases of $\mathrm{TB}$, rather than the outcome of recent transmission within the Brussels-Capital Region. This suggestion is further supported by the diversity of strains, which often correlates with the reported clonal types of the respective countries of origin of the patients [22]. Consequently, the influx of foreigners from high-TB incidence areas to the Brussels-Capital Region justifies the ongoing modifications of the existing measures in TB control policy. These modifications include better access to drugs and healthcare facilities for high-risk groups comprising underprivileged persons and asylum seekers. In addition, for the latter population, regular radiographical screening or Mantoux test screenings $(<5$ yrs) are now performed over a 2 -yr period after the time of arrival. However, in view of recent evaluations, this follow-up period should be extended to $\geqslant 5$ yrs $[23,24]$.

The present study has some limitations. Demographic data proved to be insufficient to determine links for 81 patients and contact tracing was not performed. Strain-clustering could partly result from coincidental reactivation of an endemic strain. However, many strain-clustered cases in other studies appear to result from casual contacts and involve complex chains of transmission or source cases outside the study period 


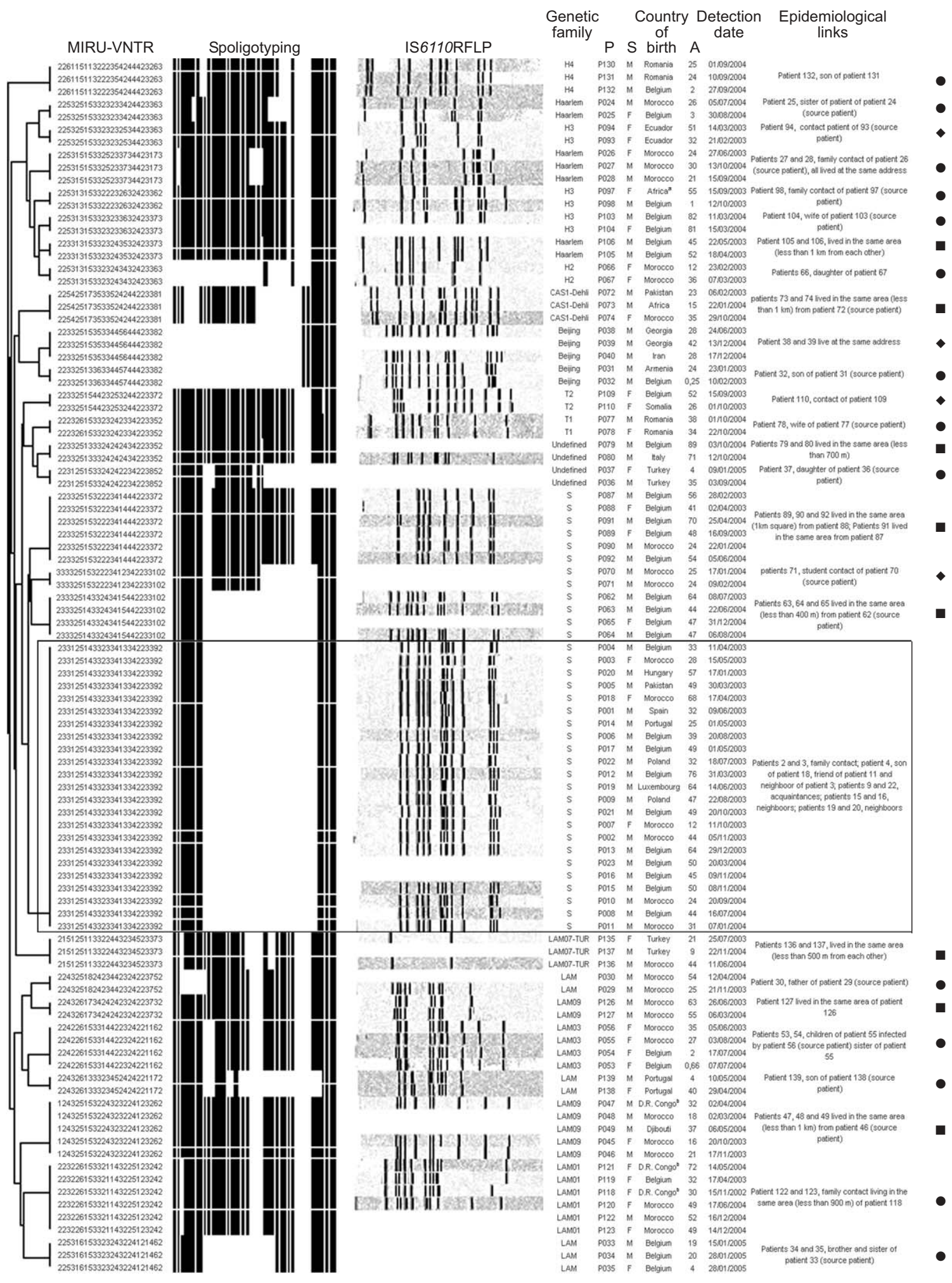

FIGURE 2. Strain-clustered patients with known epidemiological links. Clustered cases shown as vertical bars on the dendrogram were determined based on identical genotypes as defined by both variable numbers of tandem repeats of mycobacterial interspersed repetitive unit (MIRU-VNTR) typing on 24 genomic loci (MIRU: 2, 4, 10, 16, 20, 23, 24, 26, 27, 31, 39, 40; VNTR: 0424, 0577, 1955, 2163b, 2165, 2347, 2401, 2461, 3171, 3690, 4052, 4156) and spoligotyping. IS6110-restriction fragment length polymorphism patterns were additionally used for identification of strain genetic families. The large cluster corresponding to the S-Brussels strain is boxed. Sex (S), country of birth, age (A) and detection date of tuberculosis patients is given. Types of epidemiological links are represented by the following symbols. $\bullet$ : family link; patients living in a single area. P: patient; M: male; F: female. 
or the study area $[6,7,20,25]$. Intensive epidemiological investigation must be combined with rapid genotyping techniques in order to better identify such links, thereby facilitating the implementation of appropriate control measures [26]. It is noteworthy that in the present study, recent contact $(<2$ yrs) with a TB patient was noticed by initial classical epidemiological surveillance only for $\sim 20 \%$ of the strain-clustered patients for whom this information was available. Along the same lines, most transmission links (except some familial cases) identified by retrospective molecular guided contact tracing in the S-Brussels outbreak (discussed previously) remained unnoticed by initial conventional epidemiological surveillance. As noticed earlier, these observations further highlight limitations of conventional epidemiological surveillance [20] and, at the same time, emphasise the benefit of genotyping as a complementary tool for TB control.

Transmission still accounts for $20 \%$ of the cases, underlining the need for ongoing tuberculosis control. Nonetheless, the present study indicates that tuberculosis in the BrusselsCapital Region is now mainly an "imported" disease. Most cases among foreign-born patients are probably caused by infection in the country of origin, underlining the relevance of global tuberculosis control. Consistent with this hypothesis, strain clustering was less common in unsettled residents $(13.3 \%)$ as compared with settled foreign-born subjects $(34.4 \% ; \mathrm{p}<0.001)$. Cross-national transmission was limited and primarily suspected to occur from Belgian-born to foreign-settled patients, or within the foreign community. Control policies should encompass reorganised close coordination between clinicians, diagnostic laboratories and public healthcare workers, to address this complex epidemiological situation. In the present study, which represents the first largescale use of a recently standardised, fast and high-resolution PCR-based typing method [15], it was found that systematic Mycobacterium tuberculosis DNA fingerprinting was no longer the limiting step for prospective monitoring and early control of tuberculosis transmission. With this method, classical risk factors for tuberculosis transmission were identified, but its potential for more efficient intervention and prevention could not be exploited, due to strong limitations in classical epidemiological investigations and subsequent interventions. Therefore, it is only when implemented in an integrated public health system that such a real-time molecular typing method will most efficiently contribute to identify and prevent chains of transmission.

\section{ACKNOWLEDGEMENTS}

The authors would like to thank P. Sonck for his technical assistance in IS6110-RFLP and the nurses from BELTA (Brussels, Belgium) for epidemiological investigation. The authors would also like to thank A. Dediste and Ms Lafontaine (Saint-Pierre Hospital, Brussles, Belgium), O. Denis (Erasme Hospital, Brussels), M. Delmee and J. Christael-Degraux (Saint-Luc Hospital, Brussels), B. Mulongo and M. Wegge ( Saint-Jean Hospital, Brussels), D. Pierard and M. Seghers (AZ-VUB hospital, Brussels) for their kind and helpful collaboration, by providing positive cultures and collecting some patient data. This study would not have been possible without their collaboration.

\section{REFERENCES}

1 Fonds des Affections Respiratoires. Registre belge de la tuberculose 2003 [Belgium tuberculosis registry 2003]. www.fares.be/affections_respiratoires/tbc/brochure.php Date last updated: February 2005. Date last accessed: February 27, 2007.

2 Fonds des Affections Respiratoires. Registre belge de la tuberculose 2004 [Belgium tuberculosis registry 2004]. www.fares.be/affections_respiratoires/tbc/brochure.php Date last updated: March 2006. Date last accessed: February 27, 2007.

3 Ministère de la Région de Bruxelles-Capitale. Indicateur statistiques de la Région de Bruxelles-Capitales [Statistical indicators from the Brussels-Capital Region]. Brussels, Ministère de la Région de Bruxelles-Capitale, 2005.

4 Henau A. La démographie récente de la Région de Bruxelles-Capitale [Recent demography of the BrusselsCapital Region]. Brussels, Ministère de la Région de Bruxelles-Capitale, 2005.

5 Small PM, Hopewell PC, Singh SP, et al. The epidemiology of tuberculosis in San Francisco. A population-based study using conventional and molecular methods. N Engl J Med 1994; 330: 1703-1709.

6 Diel R, Schneider S, Meywald-Walter K, Ruf CM, RuschGerdes S, Niemann S. Epidemiology of tuberculosis in Hamburg, Germany: long-term population-based analysis applying classical and molecular epidemiological techniques. J Clin Microbiol 2002; 40: 532-539.

7 Ellis BA, Crawford JT, Braden CR, McNabb SJ, Moore M, Kammerer S. Molecular epidemiology of tuberculosis in a sentinel surveillance population. Emerg Infect Dis 2002; 8: 1197-1209.

8 Geng E, Kreiswirth B, Driver C, et al. Changes in the transmission of tuberculosis in New York City from 1990 to 1999. N Engl J Med 2002; 346: 1453-1458.

9 Clark CM, Driver CR, Munsiff SS, et al. Universal genotyping in tuberculosis control program, New York City, 2001-2003. Emerg Infect Dis 2006; 12: 719-724.

10 Borgdorff MW, van der Werf MJ, de Haas PE, Kremer K, van Soolingen D. Tuberculosis elimination in the Netherlands. Emerg Infect Dis 2005; 11: 597-602.

11 van Embden JD, Cave MD, Crawford JT, et al. Strain identification of Mycobacterium tuberculosis by DNA fingerprinting: recommendations for a standardized methodology. J Clin Microbiol 1993; 31: 406-409.

12 Kamerbeek J, Schouls L, Kolk A, et al. Simultaneous detection and strain differentiation of Mycobacterium tuberculosis for diagnosis and epidemiology. J Clin Microbiol 1997; 35: 907-914.

13 Mazars E, Lesjean S, Banuls AL, et al. High-resolution minisatellite-based typing as a portable approach to global analysis of Mycobacterium tuberculosis molecular epidemiology. Proc Natl Acad Sci USA 2001; 98: 1901-1906.

14 Cowan LS, Diem L, Monson T, et al. Evaluation of a twostep approach for large-scale, prospective genotyping of Mycobacterium tuberculosis isolates in the United States. J Clin Microbiol 2005; 43: 688-695.

15 Supply P, Allix C, Lesjean S, et al. Proposal for standardization of optimized mycobacterial interspersed repetitive unitvariable-number tandem repeat typing of Mycobacterium tuberculosis. J Clin Microbiol 2006; 44: 4498-4510. 
16 Oelemann MC, Diel R, Vatin V, et al. Assessment of an optimized mycobacterial interspersed repetitive-unitvariable-number tandem-repeat typing system combined with spoligotyping for population-based molecular epidemiology studies of tuberculosis. J Clin Microbiol 2007; 45: 691-697.

17 Hosmer D, Lemeshow S. Applied Logistic Regression. New York, J. Wiley and Sons, 1989.

18 Allix-Béguec C, Fauville-Dufaux M, Supply P. Three-year population-based evaluation of standardised Mycobacterial interspersed repetitive unit-variable number of tandem repeat typing of Mycobacterium tuberculosis. J Clin Microbiol 2008; [Epub ahead of print PMID: 18234864].

19 Filliol I, Driscoll JR, van Soolingen D, et al. Snapshot of moving and expanding clones of Mycobacterium tuberculosis and their global distribution assessed by spoligotyping in an international study. J Clin Microbiol 2003; 41: 1963-1970.

20 van Deutekom H, Hoijng SP, de Haas PE, et al. Clustered tuberculosis cases: do they represent recent transmission and can they be detected earlier? Am J Respir Crit Care Med 2004; 169: 806-810.
21 Thorson A, Diwan VK. Gender inequalities in tuberculosis: aspects of infection, notification rates, and compliance. Curr Opin Pulm Med 2001; 7: 165-169.

22 Brudey K, Driscoll JR, Rigouts L, et al. Mycobacterium tuberculosis complex genetic diversity: mining the fourth international spoligotyping database (SpolDB4) for classification, population genetics and epidemiology. BMC Microbiol 2006; 6: 23.

23 Cain KP, Haley CA, Armstrong LR, et al. Tuberculosis among foreign-born persons in the United States: achieving tuberculosis elimination. Am J Respir Crit Care Med 2007; 175: 75-79.

24 Vos AM, Meima A, Verver S, et al. High incidence of pulmonary tuberculosis persists a decade after immigration, The Netherlands. Emerg Infect Dis 2004; 10: 736-739.

25 Bennett DE, Onorato IM, Ellis BA, et al. DNA fingerprinting of Mycobacterium tuberculosis isolates from epidemiologically linked case pairs. Emerg Infect Dis 2002; 8: 1224-1229.

26 Ong A, Creasman J, Hopewell PC, et al. A molecular epidemiological assessment of extrapulmonary tuberculosis in San Francisco. Clin Infect Dis 2004; 38: 25-31. 